\title{
Measuring the contact force of a pantograph by image processing technology
}

\author{
T. Koyama ${ }^{1}$, M. Ikeda ${ }^{1}$, K. Nakamura ${ }^{2}$, S. Tabayashi ${ }^{3}$ \\ \& M. Niwakawa ${ }^{3}$ \\ ${ }^{1}$ Railway Technical Research Institute, Japan \\ ${ }^{2}$ Railway Technical Research Institute, \\ (currently at Central Japan Railway Company), Japan \\ ${ }^{3}$ Meidensha Corporation, Japan
}

\begin{abstract}
A contact force between pantograph and contact line is one of the important criteria to evaluate contact performance. Hence, contact force measuring methods were developed. The currently-used measurement methods use built-in sensors on the panhead. However, these sensors affected the dynamic characteristics of the pantograph. In addition, the methods need data transmitting equipment, because built-in sensors are set under a high-voltage condition. To eliminate these drawbacks, the authors have developed a new contact force measuring method which can replace the all built-in sensors with image processing sensors mounted on the rooftop of the train. To verify measurement accuracy of this method, we performed an excitation test of the pantograph. Experimental results show that the measurement method meets the requirements of the EN standard.

Keywords: contact force, pantograph, catenary, image processing, line sensor camera, EN standard.
\end{abstract}

\section{Introduction}

The contact force between a pantograph and contact line is one of the important criteria to evaluate contact performance $[1,2]$. These days, many studies have been conducted to diagnose overhead catenary system conditions by utilizing contact force measurements [3]. Hence, contact force measuring methods were developed [4-7]. 
In the currently-used methods, the contact force is measured by using built-in sensors such as loadcells and accelerometers set in a panhead. These methods, however, have some serious problems. That is, these sensors affect the dynamic characteristics of the pantograph. In addition, the method requires data transmitting equipment, because these sensors are set under a high-voltage condition.

To eliminate these drawbacks, authors have developed a new contact force measuring method, which can replace the whole of the built-in sensors with image processing sensors.

\section{The principles of the contact force measurement method}

\subsection{An outline of the contact force measurement method}

Some contact force measurement methods have already been developed. Since many of them are based on the force equilibrium equation, its outline is described in this section [6].

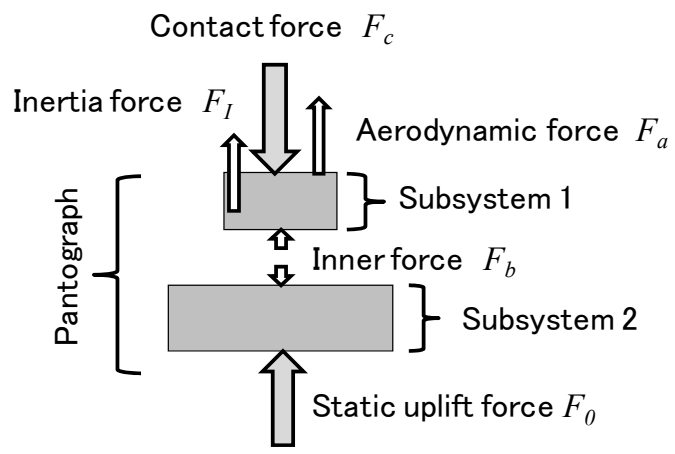

Figure 1: $\quad$ Force equilibrium in a pantograph.

Figure 1 shows a mechanical model of the pantograph. The pantograph is divided into two subsystems: the subsystem 1 corresponds to a panhead, and the subsystem 2 corresponds to an articulated frame and panhead support. Contact force $F_{c}$, inner force $F_{b}$ acting upon the interface between subsystem 1 and subsystem 2, aerodynamic force $F_{a}$ and inertia force $F_{I}$ affecting on Subsystem 1 equilibrate. Thus, we can obtain the force equilibrium equation of subsystem 1.

Eqn. (1) indicates that contact force $F_{c}$ can be evaluated as a summation of $F_{b}$, $F_{a}$, and $F_{I}$.

There is, however, no practical method for measuring aerodynamic force $F_{a}$, while the pantograph is sliding with contact wire. Since the aerodynamic force $F_{a}$ is proportional to the square of relative flow velocity $V_{t}$ against the pantograph, $F_{a}$ can be estimated by eqn. (2).

$$
F_{a}=C_{a} V_{t}^{2}
$$


where, $C_{a}$ is the proportionality constant, which can be obtained by a wind tunnel test.

Hence, only inner force $F_{b}$ and inertia force $F_{I}$ affecting subsystem 1 should be measured in order to evaluate the contact force.

\subsection{Measurement method of the inner force}

The inner force $F_{b}$ consist of spring reaction forces $F_{b s}$ of the panhead supporting springs if the friction force between the panhead and the panhead support can be negligible (See Figure 2). Therefore, the inner force $F_{b}$ can be expressed as follows:

$$
F_{b}=\sum_{i=1}^{2} F_{b s, i} \quad(i=1,2)
$$

In the case of the currently-used contact force measurement method, the inner forces $F_{b s, i}$ are generally measured by built-in sensors such as loadcells or strain gauges set in the panhead $[5,6]$.

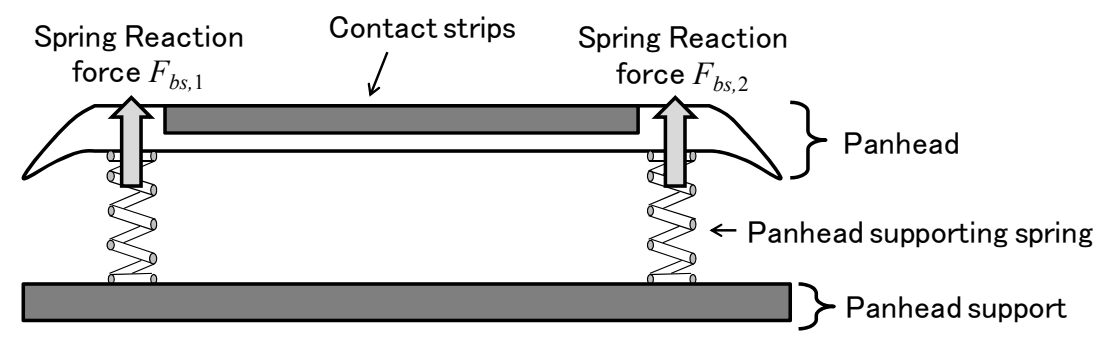

Figure 2: $\quad$ The details of inner force.

\subsection{Measurement method of the inertia force}

Only in low frequency range, the inertia force $F_{I}$ can be evaluated by mass of the panhead and the acceleration $a$ of the panhead.

$$
F_{I}=m a
$$

If the contact force is required to be measured up to high frequency range, the inertia force has to be evaluated by the product of the accelerations of plural positions on the panhead and the corresponding equivalent mass $m_{i}$ [6].

$$
F_{I}=\sum m_{i} a_{i}
$$

In the case of the currently-used contact force measurement method, the accelerations are generally measured by built-in accelerometers set into the panhead $[5,6]$. 


\section{Measuring the contact force by image processing technology}

The authors have developed a new contact force measurement method using the image processing technology with line sensor cameras, by which any built-in sensor is not needed. This chapter explains principle of this method.

\subsection{The principle of the new method}

As noted in chapter 2, if the friction force between the panhead and the panhead support can be negligible, only the spring reaction forces $F_{b s}$ and the inertia force $F_{I}$ have to be measured to obtain the contact force $F_{c}$. According to Hooke's law, the reaction forces $F_{b s}$ are obtained by the product of the spring coefficient and the spring deformation which corresponds to the relative displacement between the panhead and the panhead support. The inertia force $F_{I}$ can be obtained by the product of the mass of the panhead and the acceleration which can be obtained as the second derivative of the displacement of the panhead with respect to time. This means that we can measure the contact force by evaluating displacement of the panhead and panhead support.

\subsection{The displacements measurement method by image processing technology}

A schematic drawing of this method is as shown in Figure 3. Some markers with a black stripe are put on the surface of the panhead and the panhead support to evaluate displacements of the panhead and the panhead support. Images of these

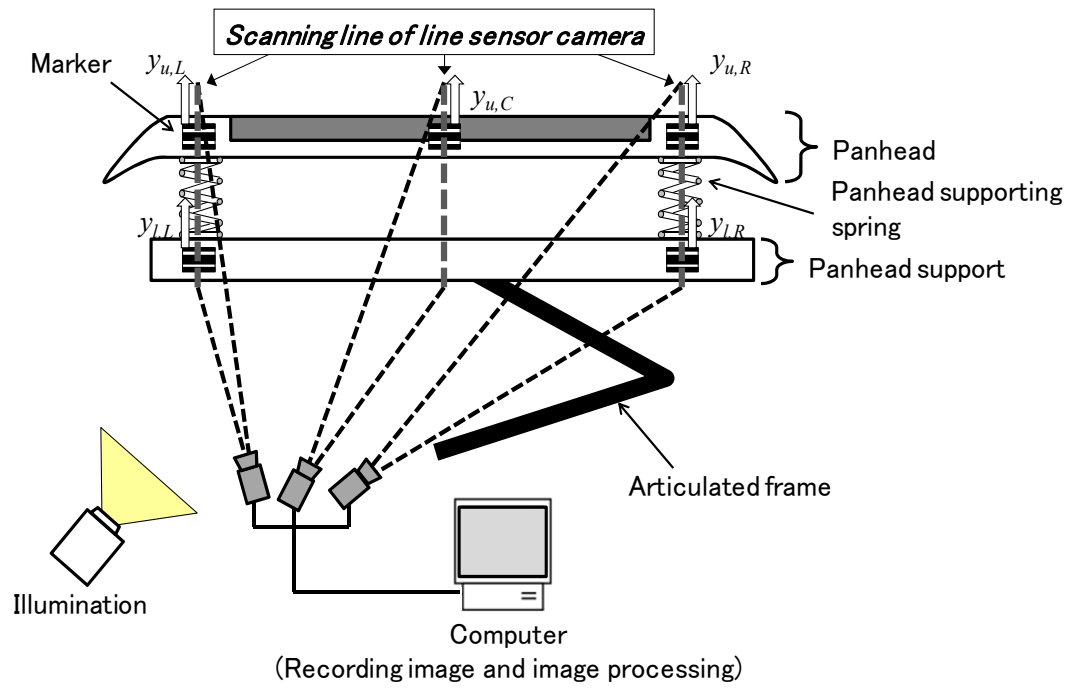

Figure 3: Measuring the contact force of pantograph by image processing technology. 
markers are taken by line sensor cameras with high time and spatial resolution mounted on the rooftop of the train near the pantograph. Illuminations installed near the pantograph help cameras to take clear images of the markers. Figure 4 shows a sample of image of the marker taken by the line sensor camera. This image consists of many time-ordered one-dimensional images captured by the line sensor camera.

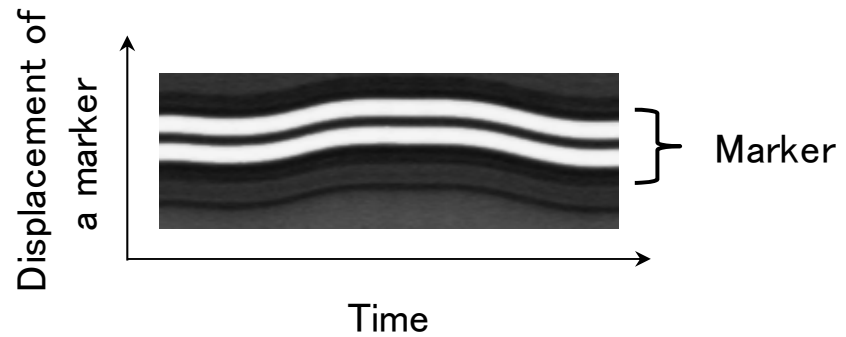

Figure 4: Images of a marker taken by a line sensor camera.

Symbols, $y_{u, L}, y_{l, L}, y_{u, R}, y_{l, R}$ and $y_{u, C}$ in the Figure 3 are vertical displacements of the markers. These displacements are obtained by the line sensor cameras using image processing technology with the pattern matching based on the normalized cross-correlation (NCC) method. NCC enhanced spatial resolution of displacement measurement. Figure 5 shows the displacement evaluated with/without NCC. Figure 5 indicates that the spatial resolution can be improved by using NCC.

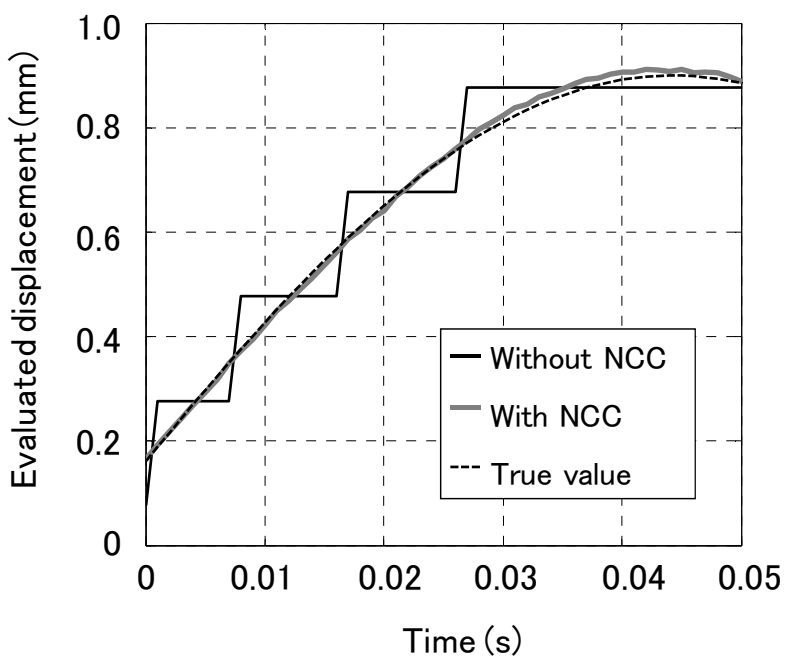

Figure 5: Displacement of a marker evaluated by NCC. 


\subsection{Measuring the contact force}

As mentioned before, the spring reaction forces $F_{b s}$ of the panhead supporting spring can be evaluated by the product of the spring coefficient and the spring deformation. The spring deformation can be taken by the image processing technologies as described in section 3.1. If the friction force between the panhead and the panhead support can be negligible, the inner force $F_{b}$ consists of only spring reaction forces $F_{b s}$.

Vertical accelerations $a_{u, L}, a_{u, R}$ and $a_{u, C}$ at the position of the markers can be obtained as the second derivatives of the displacements $y_{u, L}, y_{u, R}$ and $y_{u, C}$ with respect to time. The inertia force $F_{I}$ acting on the panhead is obtained by summing the products of the accelerations and the corresponding equivalent masses. The method for identification of the equivalent masses is described in the following.

The equivalent masses can be obtained by an excitation test. The inertia force $F_{I}$ can be estimated by eqn. (6):

$$
F_{I}=F_{c, \text { applied }}-F_{b}
$$

where, $F_{c, \text { applied }}$ and $F_{b}$ are excitation force and inner force, respectively. A frequency transfer function of $a_{u, i}(i=\mathrm{L}, \mathrm{C}, \mathrm{R})$ versus $F_{I}$ is expressed as follows:

$$
G_{i}(j \omega)=\hat{a}_{u, i} / \hat{F}_{I}
$$

where, $j$ and $\omega$ are imaginary unit and angular frequency. $\hat{a}_{u, i}$ indicates the Fourier transform of $a_{u, i}$. The frequency transform function can be written in matrix form:

$$
\mathbf{G}=\left(\begin{array}{ccc}
G_{L}\left(j \omega_{1}\right) & G_{C}\left(j \omega_{1}\right) & G_{R}\left(j \omega_{1}\right) \\
\vdots & \\
G_{L}\left(j \omega_{n}\right) & G_{C}\left(j \omega_{n}\right) & G_{R}\left(j \omega_{n}\right)
\end{array}\right)
$$

By using eqn. (8), the equivalent masses $m_{L}, m_{C}$ and $m_{R}$ for each marker are calculated as follows:

$$
\left(\begin{array}{lll}
m_{L} & m_{C} & m_{R}
\end{array}\right)^{T}=\left(\begin{array}{c}
\operatorname{Re}(\mathbf{G}) \\
\operatorname{Im}(\mathbf{G})
\end{array}\right)^{+} \mathbf{V}
$$

where, $\operatorname{Re}(\mathbf{G})$ and $\operatorname{Im}(\mathbf{G})$ indicate real part and imaginary part of $\mathbf{G}$, respectively. The superscript $T$ and + indicates transposed matrix and generalized inverse matrix, respectively. A vector $\mathbf{V}$ is expressed as follows:

$$
\begin{aligned}
\mathbf{V} & =\left(\begin{array}{llllll}
v_{1} & \cdots & v_{n} & v_{n+1} & \cdots & v_{2 n}
\end{array}\right)^{T} \\
v_{1} & =\cdots=v_{n}=1, v_{n+1}=\cdots=v_{2 n}=0
\end{aligned}
$$

As stated above, we can evaluate the inner force and the inertia force required to measure the contact force by using the image processing technology with line 
sensor cameras. Therefore, the contact force can be measured by substituting these forces and aerodynamic force in eqn. (1). Figure 6 indicates the measurement procedure of this method.

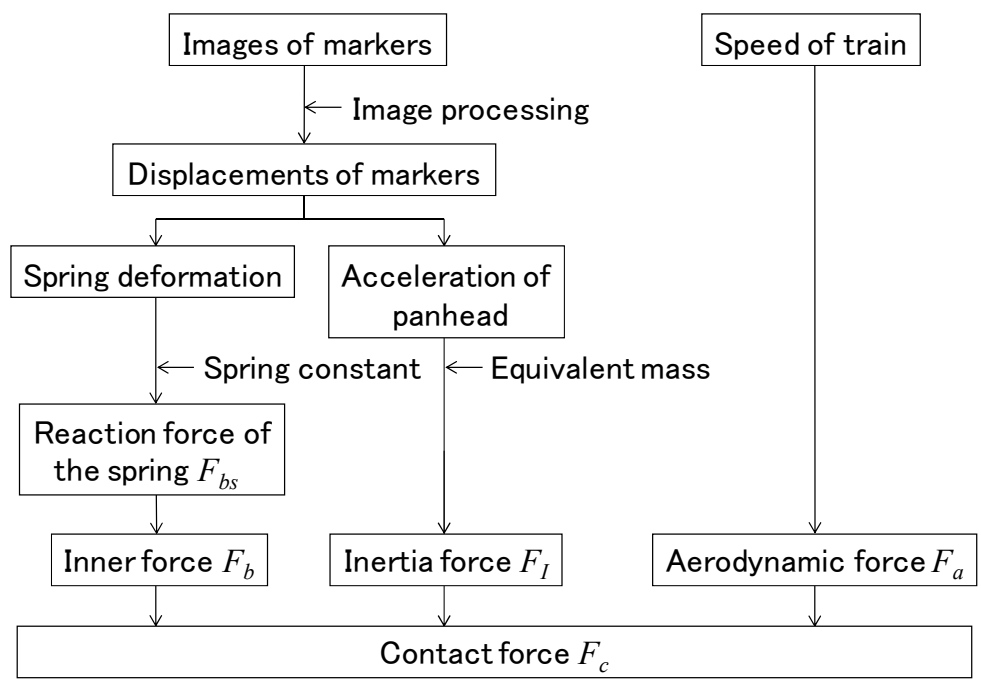

Figure 6: Procedure of the measurement method of the contact force by image processing technology.

\section{Verification test of the new method}

Authors carried out an excitation test of a pantograph in order to verify measurement accuracy of the new method. Figure 7 shows photo of the test.

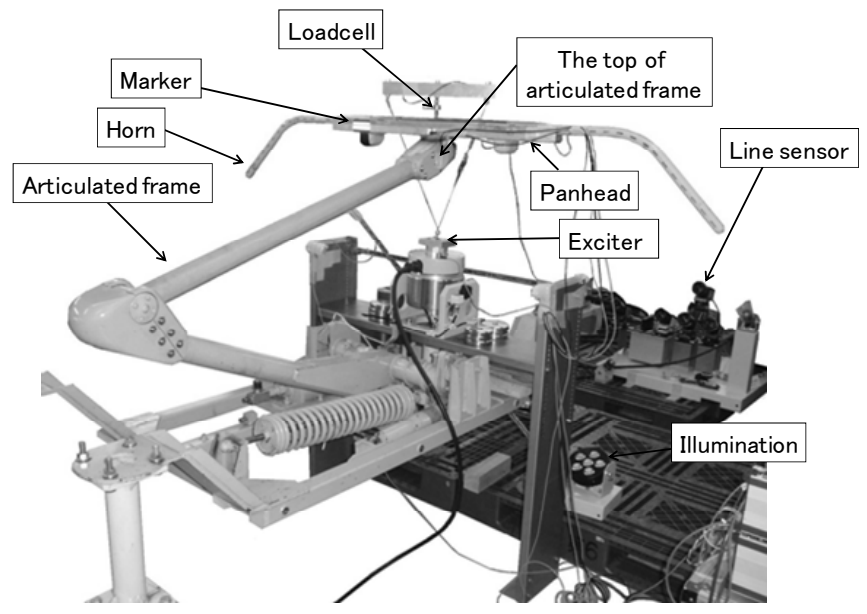

Figure 7: Photo of the excitation test of a pantograph. 
A single-arm type pantograph was used in the test. Friction force, which affected between the panhead and the panhead support of this pantograph, can be negligible.

An exciter was placed under the panhead and a loadcell was put on the panhead to measure excitation force $F_{c \text {,applied }}$. The excitation force $F_{c, \text { applied }}$ was compared with measured force $F_{c \text {,measured }}$ measured by the new method, which was identical to the excitation force ideally.

Two line sensor cameras (pixels: 7450pix, sampling frequency: $4.73 \mathrm{kHz}$ ) and illuminations were mounted near the pantograph. Three markers were set on the surfaces of the panhead and the panhead support (see Figure 8). One line sensor camera took images of two markers put on the surface of the panhead and the pantograph support to evaluate the spring reaction force of the panhead supporting springs and the inertia force of the panhead. The other line sensor camera took images of a marker put on the surface of the panhead to evaluate the inertia force of the panhead.

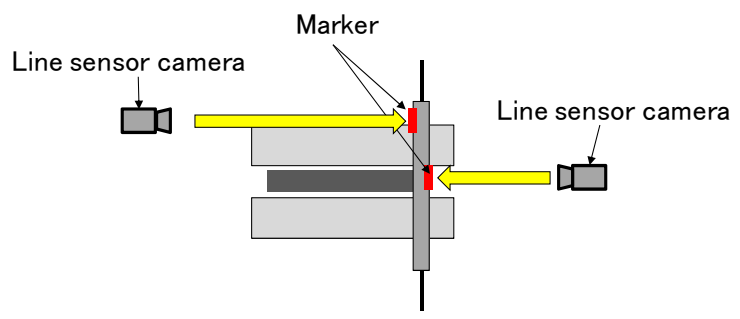

(a) overview

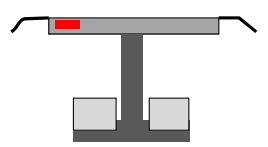

(b) side view

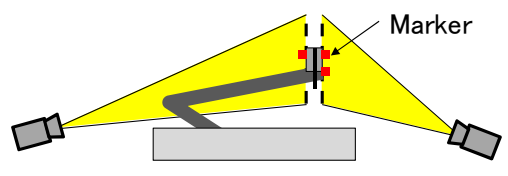

(c) front view

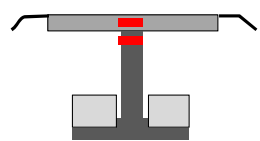

(d) side view

Figure 8: The position of line sensor cameras.

The test result is as shown in Figure 9. This figure indicates gain and phase of the frequency transfer function of the measured contact force $F_{c \text {,estimated }}$ versus the exciting force $F_{c, \text { applied }}$.

The European Standard (EN50317) provides the allowance of the measurement accuracy of a contact force measurement method calculated by using following formula [8]:

$$
\left\{1-\frac{1}{\left(f_{n}-f_{1}\right)} \sum_{k=1}^{n-1}\left(f_{k+1}-f_{k}\right)\left|1-\frac{\hat{F}_{c, \text { measured }}}{\hat{F}_{c, \text { applied }}}\right|\right\} \times 100 \%
$$


where, $f_{n}$ and $f_{l}$ is the maximum frequency and minimum frequency of the frequency range required to measure the contact force. We verified the measurement accuracy of the new method by using eqn. (11). Table 1 shows the accuracy of the new method and the accuracy required on the standard. It can be confirmed that the new contact force measurement method has satisfactory measurement accuracy, which meets a requirement of the standard.

Table 1: $\quad$ The measurement accuracy of the new method.

\begin{tabular}{|l|c|c|}
\hline \multirow{2}{*}{} & \multicolumn{2}{|c|}{ Measurement accuracy \% } \\
\cline { 2 - 3 } & $\begin{array}{l}\text { Measurement accuracy } \\
\text { of our method }\end{array}$ & $\begin{array}{l}\text { Required accuracy } \\
\text { on EN50317 }\end{array}$ \\
\hline Up to $10 \mathrm{~Hz}$ & 91.5 & $>80$ \\
\hline Up to $20 \mathrm{~Hz}$ & 90.1 & $>90$ \\
\hline
\end{tabular}

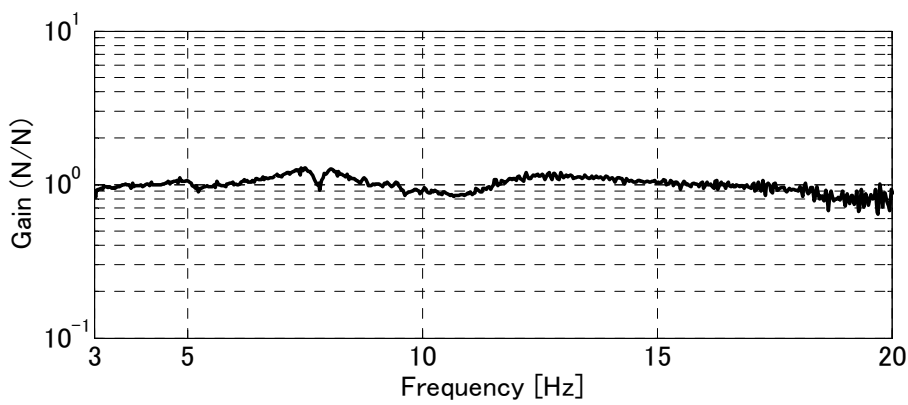

(a) Gain of transfer function $\hat{F}_{c \text {,measured }} / \hat{F}_{c \text {,applied }}$

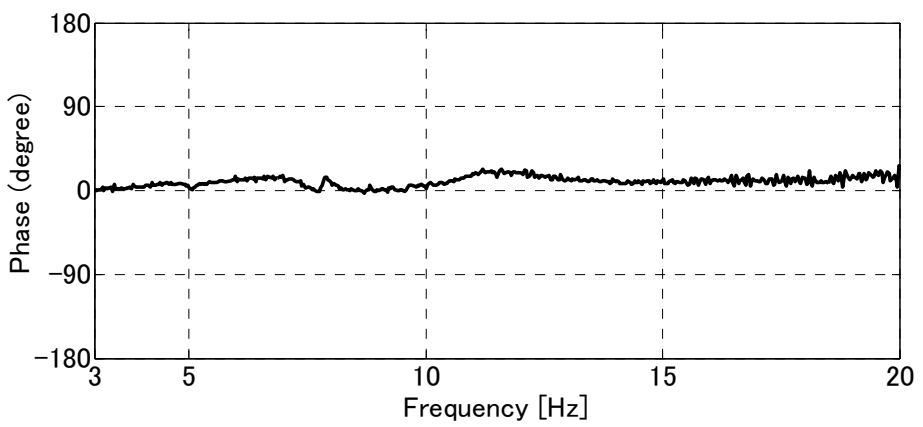

(b) Phase of transfer function $\hat{F}_{c, \text { measured }} / \hat{F}_{c \text {,applied }}$

Figure 9: The result of vilification test. 


\section{Conclusions}

The authors have developed the new contact force measurement method using the image processing technology, which needs no built-in sensor such as loadcells and so on. In order to verify measurement accuracy of this method, the excitation test was carried out. Consequently, it can be confirmed that the contact force measurement method has the satisfactory measurement accuracy, which meets requirement of EN50317.

\section{References}

[1] EN 50119:2001, Railway applications - Fixed installations - Electric traction overhead contact lines, CENELEC, 2001.

[2] EN 50367:2002, Railway applications - Current collection systems Technical criteria for the interaction between pantograph and overhead line (to achieve free access), CENELEC, 2002.

[3] Shunichi K., Takahiro F., Kazuyoshi N., Diagnosis of Overhead Contact Line based on Contact Force, Quarterly Report of RTRI, Vol.47, No.1, pp. 39-45, 2006.

[4] Mitsuru I., Sei N., Takayuki U., A precise contact force measuring method for overhead catenary system, WCRR 2001, 2001.

[5] Sung-Il S., Young-Hyun C., Jin-Yong M., Choon-Soo P., Kin-Hwan K., A Study on the Measurement of Contact Force of Pantograph on High Speed Train, ICCAS2005, Korea, 2005.

[6] Mitsuru I., A study on the method of measuring the contact force between pantograph and contact wire (1st report, Extension of the frequency range for contact force measurement by an improved method of evaluating), Transactions of the Japan Society of Mechanical Engineers, Ser. C, Vol. 70, No.694, 2004 (in Japanese).

[7] Mitsuru I., The Contact Force between Pantograph and Contact Wire -An Estimation Method Using the Inversion Technique-, Quarterly Report of RTRI, Vol.45, No.2, pp. 80-85, 2004.

[8] EN 50317:2002, Railway applications - Current collection systems Requirement for and validation of measurements of the dynamic interaction between pantograph and overhead contact line, CENELEC, 2002. 